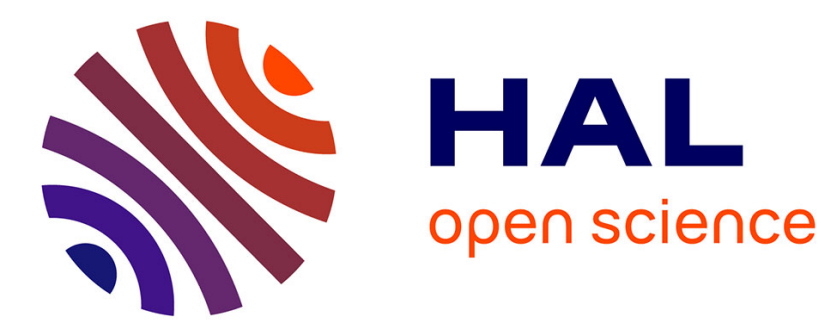

\title{
Giant resonant radiative heat transfer between nanoparticles
}

\author{
Yong Zhang, Hing-Liang Yi, He-Ping Tan, Mauro Antezza
}

\section{To cite this version:}

Yong Zhang, Hing-Liang Yi, He-Ping Tan, Mauro Antezza. Giant resonant radiative heat transfer between nanoparticles. Physical Review B, 2019, 100, pp.134305. 10.1103/PhysRevB.100.134305 . hal-02317225

\section{HAL Id: hal-02317225 \\ https://hal.science/hal-02317225}

Submitted on 14 Oct 2020

HAL is a multi-disciplinary open access archive for the deposit and dissemination of scientific research documents, whether they are published or not. The documents may come from teaching and research institutions in France or abroad, or from public or private research centers.
L'archive ouverte pluridisciplinaire HAL, est destinée au dépôt et à la diffusion de documents scientifiques de niveau recherche, publiés ou non, émanant des établissements d'enseignement et de recherche français ou étrangers, des laboratoires publics ou privés. 


\title{
Giant resonant radiative heat transfer between nanoparticles
}

\author{
Yong Zhang, ${ }^{1,2}$ Hong-Liang Yi $\odot,{ }^{1,2, *}$ He-Ping Tan, ${ }^{1,2}$ and Mauro Antezza ${ }^{3,4, \dagger}$ \\ ${ }^{1}$ School of Energy Science and Engineering, Harbin Institute of Technology, Harbin 150001, People's Republic of China \\ ${ }^{2}$ Key Laboratory of Aerospace Thermophysics, Ministry of Industry and Information Technology, Harbin 150001, People's Republic of China \\ ${ }^{3}$ Laboratoire Charles Coulomb (L2C), UMR 5221 CNRS-Université de Montpellier, F- 34095 Montpellier, France \\ ${ }^{4}$ Institut Universitaire de France, 1 Rue Descartes, F-75231 Paris, France
}

(Received 18 July 2019; revised manuscript received 30 September 2019; published 15 October 2019)

\begin{abstract}
Near-field radiative heat transfer exhibits various effects, such as amplification due to the geometry of the system. In this work, we construct a periodic layered structure which consists of multiple layers of alternating materials. Radiative heat transfer (RHT) between nanoparticles placed on each side of an intermediate structure is studied. Thermal energy exchange between nanoparticles is assisted by transmitted evanescent fields, which is theoretically included in the system's Green's function. We show that the resulting heat transfer with the assistance of a multilayered structure is more than five orders of magnitude higher than that in the absence of the multilayered structure at the same interparticle distance. This increase is observed over a broad range of distances ranging from near to far field. This is due to the fact that the intermediate multilayered structure supports hyperbolic phonon polaritons, where the edge frequencies of the type-I and type-II reststrahlen bands coincide at a value that is approximately equal to the nanoparticle resonance. This allows high- $k$ evanescent modes to resonate with the nanoparticles. The effects of the number of layers and fill factor in the multilayered structure on RHT are examined. Finally, we show that when there is a lateral distance between the two particles assisted by the interference of surface waves, RHT conductance exhibits an oscillating and nonmonotonic behavior with respect to the lateral distance between nanoparticles. These results illustrate a powerful method for regulating energy transport in particle systems and can be relevant for effective energy management at nano-micro scales.
\end{abstract}

DOI: 10.1103/PhysRevB.100.134305

\section{INTRODUCTION}

Since the pioneering work of Polder and Van Hove [1], it is well known that radiative heat transfer (RHT) between two objects in proximity (i.e., in the near-field regime) may significantly increase [2-8]. This is caused by tunneling of evanescent modes as surface plasmon polaritons (SPPs) or surface phonon polaritons (SPhPs) [9-19]. The huge nearfield radiative heat flux allows the use of various technologies for near-field energy conversion [20,21], data storage [22], and active thermal management [23] at the nanoscale with transistors [24,25], thermal rectifiers [26-29], and memories [30].

In the far-field regime, energy transfer between the two bodies results exclusively from propagating waves; hence it greatly decreases. However, the situation dramatically changes if a third body is introduced between the two reservoirs. For planar configurations, near-field radiative heat transfer (NFRHT) between two bodies can be amplified thanks to coupling of reservoirs to a third thin slab placed between them [31]. It has been also shown that if the interacting bodies are connected in the near field with weakly dissipating hyperbolic waveguides, one could measure super-Planckian heat transfer at a large separation distance [32]; this situation has been investigated during research on RHT between

\footnotetext{
*yihongliang@hit.edu.cn

†mauro.antezza@umontpellier.fr
}

particles. Recently, it was shown that strong enhancement of heat flux is possible due to the presence of many-body interactions [33-35]. RHT between two particles in the presence of one or two plates has received focus [36-40]. It was reported that assistance of surface wave propagation causes a significant amplification of RHT at distances ranging from near to far field. Note that the two particles investigated in [36-40] were placed on the same side of the plate, indicating that RHT amplification is attributed to surface waves reflected from the plate. Note that, in the context of Förster/resonant energy transfer [41], transmission configurations where the two dipoles are placed on each side of a slab with finite thickness were investigated theoretically and experimentally $[42,43]$. Several strategies were suggested to tune RHT, and different multilayered structures were extensively studied for this purpose. Indeed, in multilayer structures, the interactions between surface polaritons at multiple interfaces exhibit exotic features, including near-field thermal radiation tuning [44-47].

In this work, we focus on RHT between $\mathrm{SiC}$ nanoparticles when they are placed on each side of a multilayered structure. We constructed a periodic layered structure consisting of alternating $\mathrm{SiC}$ and $\mathrm{NaBr}$ films. The structure supports hyperbolic $\mathrm{SPhPs}$, and the edge frequencies of the type-I and type-II reststrahlen bands coincide at a value that is approximately equal to the nanoparticle resonance. We show that the intermediate multilayered structure couples many high- $k$ modes to RHT between nanoparticles, generating a giant resonant enhancement in RHT at long distances. Moreover, we find that 


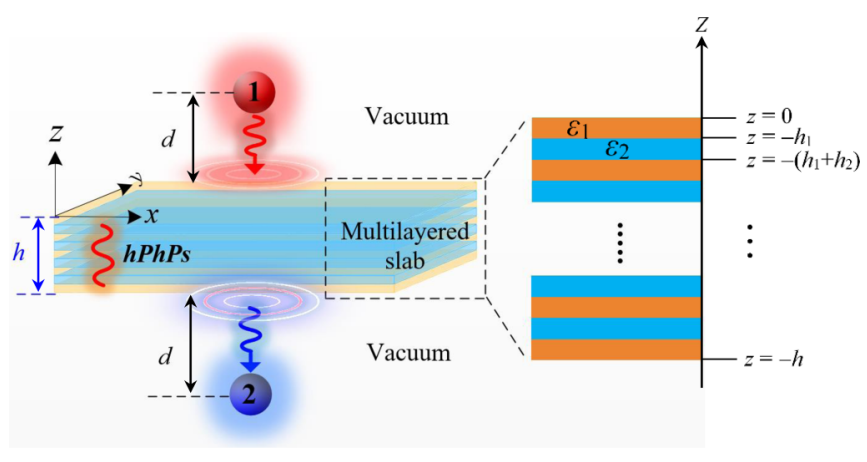

FIG. 1. Schematic of RHT between two nanoparticles in the presence of a multilayered slab. The nanoparticles lie on opposite sides of the intermediate structure with thickness $h$. The top surface of the slab coincides with the origin. The periodic multilayered slab consists of multiple layers of alternating materials.

RHT between particles exhibits an oscillating behavior when the propagating waveguides of the intermediated multilayered structure start to play a role.

The paper is structured as follows. In Sec. II, we introduce the geometry of our system, define the Green's function in the presence of the intermediate multilayered structure, and derive the heat flux between two nanoparticles. Section III introduces the optical properties of the multilayered structure. The giant resonant enhancement in RHT between nanoparticles is discussed in Sec. IV. An oscillating RHT is observed when the two nanoparticles are separated by a lateral distance, and this behavior is analyzed in Sec. V. Conclusions are offered in Sec. VI.

\section{THEORETICAL ASPECTS}

To start, let us consider RHT between particle 1 and particle 2, as shown in Fig. 1. This model contains two isotropic spherical particles with radius $R=5 \mathrm{~nm}$ as simple radiating dipoles, which is valid when the center-to-center distance $L>$ $3 R$ [36-40]. We insert a periodic layered structure consisting of multiple layers of alternating materials (film 1 and film 2) between the two nanoparticles. Films 1 and 2 are characterized by their respective dielectric functions $\varepsilon_{1}$ and $\varepsilon_{2}$. Each material has an individual thickness $h_{i}(i=1,2)$. If we have $N$ layers, the total thickness of the complete multilayered structure is $h=\left[(N+1) h_{1}+(N-1) h_{2}\right] / 2$. The coordinate system is defined such that the $x-y$ plane coincides with the surface of the topmost layer, and the $z$ direction is orthogonal to the surface. Both nanoparticles are separated from the surface by a distance of $d$. The vertical positions of the top and bottom particles are $z_{1}=d$ and $z_{2}=-h-d$, respectively. Note that we have omitted any magnetic dipole contribution, which is much smaller than the electric dipole contribution, and higher-order multipoles are neglected.

We note that, by using external reservoirs, particles 1 and 2 , as well as the multilayered structure, could be kept at fixed temperatures $T_{1}, T_{2}$, and $T_{S}$, respectively. We assume now that the temperature of the entire system is set to $T$, while the temperature of particle 1 is slightly increased to $T_{1}=T+\Delta T$. Now, because particle 2 has no net heat exchange with the multilayered structure (both are still at the same temperature
$T_{2}=T_{S}=T$ ), particle 2 only exchanges heat with particle 1 . The RHT conductance $\Phi$ between these two particles can be determined as the temperature difference $\Delta T$ tends to 0 . This is the quantity we discuss in the paper, with a particular focus on how the presence of the multilayered structure modifies $\Phi$. We note that particle 1 will also exchange a portion of its heat with the multilayered structure exclusively, and this heat transfer will not affect the energy transferred from particle 1 to particle 2 . Thus, in this work, the intermediate structure purely acts as an electromagnetic signal amplifier or attenuator, modifying the way in which direct heat exchange occurs between particles in vacuum. The RHT conductance $\Phi$ between two identical nanoparticles at a temperature $T$ can be conveniently expressed in terms of the following Green's function (GF) [37]:

$$
\Phi=4 \int_{0}^{+\infty} \frac{d \omega}{2 \pi} \hbar \omega n^{\prime}(\omega, T) k_{0}^{4}[\operatorname{Im}(\alpha)]^{2} \operatorname{Tr}\left[\mathbb{G} \mathbb{G}^{*}\right],
$$

where $k_{0}=\omega / c$ and $\alpha$ are the free-space wave number and the particle's electric frequency-dependent polarizability, respectively. In the limit $R \ll \delta$ (where $\delta$ is the skin depth of the given material), $\alpha$ is written in the well-known ClausiusMossotti form, $\alpha(\omega)=4 \pi R^{3}\{[\varepsilon(\omega)-1] /[\varepsilon(\omega)+2]\}$, where $\varepsilon(\omega)$ is the electric permittivity of the particle. $n^{\prime}(\omega, T)$ denotes the derivative with respect to $T$ of the Bose-Einstein distribution $n(\omega, T)=\left[\exp \left(\hbar \omega / k_{B} T\right)-1\right]^{-1}$, and $\mathbb{G}$ denotes the GF. For two isolates particles, the free-space GF is

$$
\mathbb{G}=\mathbf{G}_{0}=\frac{e^{i k_{0} L}}{4 \pi k_{0}^{2} L^{3}}\left(\begin{array}{ccc}
a & 0 & 0 \\
0 & b & 0 \\
0 & 0 & b
\end{array}\right),
$$

where $a=2-2 i k_{0} L$ and $b=k_{0}^{2} L^{2}+i k_{0} L-1$. In the presence of an intermediate multilayered structure, the transmitted electric field in the lower half-space is modified by the slab. The following transmitted GF $[41,48]$ is used instead of $\mathbf{G}_{0}$ :

$$
\begin{aligned}
\mathbb{G} & =\mathbf{G}_{\mathrm{tr}} \\
& =\frac{i}{4 \pi} \int_{0}^{+\infty}\left[t_{s} \mathbf{M}_{\mathrm{tr}}^{s}+t_{p} \mathbf{M}_{\mathrm{tr}}^{p}\right] e^{i\left[-k_{z 0}\left(z_{2}+h\right)+k_{z 0} z_{1}+k_{x} d_{x}\right]} k_{\rho} d k_{\rho},
\end{aligned}
$$

where $k_{\rho}$ and $k_{z 0}=\sqrt{k_{0}^{2}-k_{\rho}^{2}}$ are the lateral wave number along the surface and the $z$ component of the wave vector in vacuum, respectively. $d_{x}$ is the lateral center-to-center distance between the two nanoparticles along the $x$ axis. The decaying term $e^{i\left[-k_{z 0}\left(z_{2}+h\right)+k_{z 0} z_{1}\right]}$ reveals the evanescent feature of the transmitted waves. When there is a lateral distance between the two particles $\left(d_{x} \neq 0\right)$, surface wave propagation along the $x$ axis would play a role in determining RHT. The exponentially decaying term $e^{i k_{x} d_{x}}$ quantifies evanescent surface waves along the $x$ axis. $t_{s}$ and $t_{p}$ are the Fresnel transmission coefficients of the multilayered structure associated for $s$ and $p$ polarization, respectively. The exact solution is

$$
t=\frac{t_{01} t_{12} e^{i k_{z, 1} h_{1}}}{1-r_{10} r_{12} e^{i 2 k_{z, 1} h_{1}}},
$$

where we denote 0,1 , and 2 as the vacuum region above film 1 , inside film 1 , and below film 1 , respectively. One can refer to Refs. $[44,49,50]$ for a detailed method for calculating $t$. The 

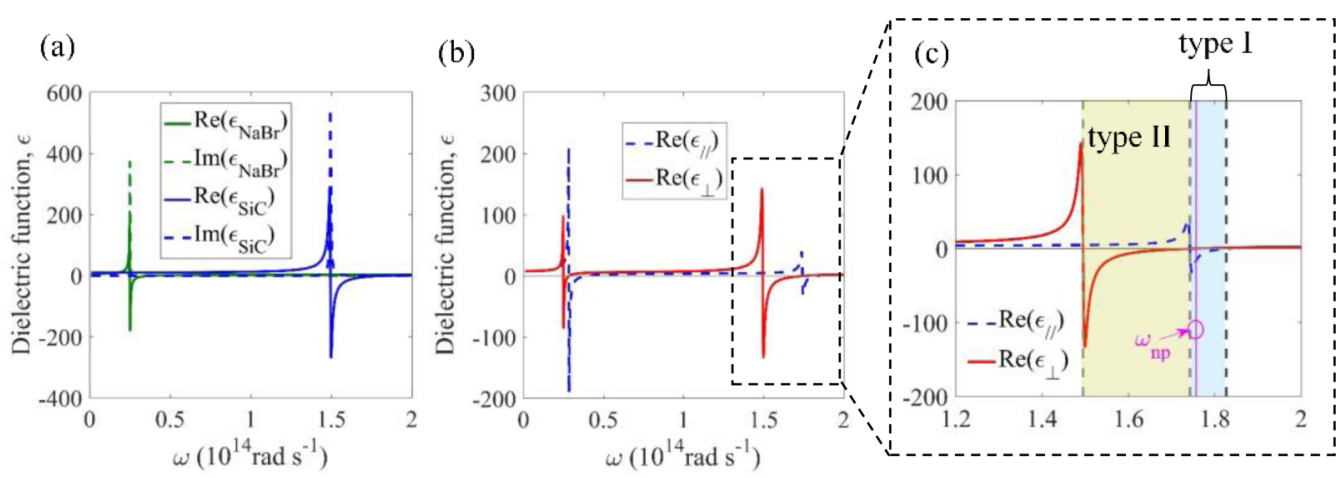

FIG. 2. Optical properties of the multilayered structure. (a) Dielectric functions of SiC and NaBr. (b) Real parts of the in-plane and out-of-plane permittivities of the multilayered structure predicted using EMA. (c) Type-I and type-II reststrahlen bands near the SiC reststrahlen band, which are shaded in blue and yellow, respectively. The resonance of the SiC nanoparticle $\omega_{n p}$ is shown in panel (c).

transmitted GF becomes the free-space GF when $t_{s}=t_{p}=1$ $[42,48]$. The transmission matrices $\mathbf{M}_{\mathrm{tr}}^{s}$ and $\mathbf{M}_{\mathrm{tr}}^{p}$ are defined as

$$
\begin{aligned}
& \mathbf{M}_{\mathrm{tr}}^{s}=\frac{1}{k_{z 1}}\left[\begin{array}{ccc}
\frac{1}{2} \mathrm{~A}_{1} & 0 & 0 \\
0 & \frac{1}{2} \mathrm{~A}_{2} & 0 \\
0 & 0 & 0
\end{array}\right], \\
& \mathbf{M}_{\mathrm{tr}}^{p}=\frac{1}{k_{0}^{2}}\left[\begin{array}{ccc}
\frac{k_{z}}{2} \mathrm{~A}_{2} & 0 & i k_{\rho} J_{1}\left(k_{\rho} d_{x}\right) \\
0 & \frac{k_{z}}{2} \mathrm{~A}_{1} & 0 \\
i k_{\rho} J_{1}\left(k_{\rho} d_{x}\right) & 0 & J_{0}\left(k_{\rho} d_{x}\right) k_{\rho}^{2} / k_{z}
\end{array}\right],
\end{aligned}
$$

where $J_{n}$ is the cylindrical Bessel function of order $n, A_{1}=$ $J_{0}\left(k_{\rho} d_{x}\right)+J_{2}\left(k_{\rho} d_{x}\right)$, and $A_{2}=J_{0}\left(k_{\rho} d_{x}\right)-J_{2}\left(k_{\rho} d_{x}\right)$. When both particles are located in the upper half-space, the GF is the sum of the free-space GF and the reflected GF $\mathbf{G}_{\text {ref }}$, as discussed in Refs. [36-40].

\section{OPTICAL PROPERTIES OF THE MULTILAYERED STRUCTURE}

We choose nanoparticles and film 1 made of $\mathrm{SiC}$, which is a typical polar dielectric material, where the dielectric function is described using the Drude-Lorentz model [51],

$$
\varepsilon(\omega)=\varepsilon_{\infty} \frac{\omega_{L}^{2}-\omega^{2}-i \Gamma \omega}{\omega_{T}^{2}-\omega^{2}-i \Gamma \omega},
$$

with high-frequency dielectric constant $\varepsilon_{\infty}=6.7$, longitudinal optical frequency $\omega_{L}=1.83 \times 10^{14} \mathrm{rad} / \mathrm{s}$, transverse optical frequency $\omega_{T}=1.49 \times 10^{14} \mathrm{rad} / \mathrm{s}$, and damping $\Gamma=$ $8.97 \times 10^{11} \mathrm{rad} / \mathrm{s}$. It is stressed that the electric polarizability predicts a nanoparticle resonance $\omega_{n p}$ corresponding to the condition $\varepsilon(\omega)+2=0$, which gives $\omega_{n p}=1.756 \times$ $10^{14} \mathrm{rad} / \mathrm{s}$ for SiC. Film 2 of the multilayered structure made is a polar dielectric $\mathrm{NaBr}$ with the following dielectric function [52]:

$$
\varepsilon_{2}(\omega)=\varepsilon_{\infty} \frac{\omega_{L}^{2}-\omega_{T}^{2}}{\omega_{T}^{2}-\omega^{2}+i \Gamma \omega},
$$

where $\varepsilon_{\infty}=2.6, \quad \omega_{L}=0.39 \times 10^{14} \mathrm{rad} / \mathrm{s}, \quad \omega_{T}=0.25 \times$ $10^{14} \mathrm{rad} / \mathrm{s}$, and $\Gamma=2.6 \times 10^{11} \mathrm{rad} / \mathrm{s}$.

We have constructed a $\mathrm{SiC} / \mathrm{NaBr}$ multilayered structure. To provide reasonable justification for our structure, we begin by discussing the optical properties of this structure. Because the periodicity of the multilayered structure is far smaller as compared to the relevant thermal wavelength at the chosen temperature, the effective medium approximation (EMA) can be used to describe the multilayered structure as a homogenous uniaxial material, where the optical axis is perpendicular to the interfaces. According to EMA, the in-plane and out-ofplane permittivities, as defined with respect to the surfaces of the layers, are approximately $\varepsilon_{\|}=f \varepsilon_{1}+(1-f) \varepsilon_{2}$ and $\varepsilon_{\perp}=$ $1 /\left(f / \varepsilon_{1}+(1-f) / \varepsilon_{2}\right)$, respectively, where $f=h_{1} /\left(h_{1}+h_{2}\right)$ is the fill factor of the $\mathrm{SiC}$ film. As a concrete example, the dielectric functions of $\mathrm{SiC}$ and $\mathrm{NaBr}$ are plotted in Fig. 2(a), and the multilayered structure obtained from EMA for $f=$ 0.5 is shown in Fig. 2(b). We see that the in-plane and outof-plane permittivities exhibit opposite signs in four spectral bands in Fig. 2(b), indicating that our structure supports hyperbolic phonon polaritons (hPhPs). One can expect that the contributions from bands near the nanoparticle resonance $\omega_{n p}$ would dominate RHT between the two SiC particles. Therefore, bands far from $\omega_{n p}$ need not be considered. Let us take a detailed look at the right reststrahlen bands near the $\mathrm{SiC}$ reststrahlen band in Fig. 2(c). This figure shows that the upper band $[1.7424-1.826] \times 10^{14} \mathrm{rad} / \mathrm{s}$ corresponds to a type-I hyperbolic response with $\operatorname{Re}\left(\varepsilon_{\|}\right)<0$ and $\operatorname{Re}\left(\varepsilon_{\perp}\right)>0$, while the lower band [1.495-1.742] $\times 10^{14} \mathrm{rad} / \mathrm{s}$ corresponds to a type-II hyperbolic response with $\operatorname{Re}\left(\varepsilon_{\|}\right)>0$ and $\operatorname{Re}\left(\varepsilon_{\perp}\right)<0$. In such a hyperbolic material with its surface normal to the $z$ axis, the hPhP waves can be restricted to a specific direction of angle $\theta=\operatorname{artan}\left(i \sqrt{\varepsilon_{\perp}} / \sqrt{\varepsilon_{\|}}\right)$with respect to the $z$ axis, resulting in extraordinary rays with very large momentum, i.e., high- $k$ modes. These modes are guided within the multilayered structure. Interestingly, these two hyperbolic bands nearly intersect at a frequency of $\omega_{h}=1.742 \times 10^{14} \mathrm{rad} / \mathrm{s}$, which is approximately equal to the $\mathrm{SiC}$ nanoparticle resonance $\omega_{n p}$.

To gain more insight on the hPhPs, we plot twodimensional (2D) contour plots of the imaginary part of the transmission coefficient $\operatorname{Im}\left(t_{p}\right)$ by EMA in Fig. 3(a) for $h=1 \mu \mathrm{m}$. Meanwhile, we plot the dispersion lines obtained from $k_{\rho}=(-\delta / h)\left\{n \pi+\operatorname{artan}\left[\omega /\left(\delta \omega \varepsilon_{\perp}\right)\right]+\operatorname{artan}\left[1 /\left(\delta \varepsilon_{\perp}\right)\right]\right\}$, in which $\delta=-i \sqrt{\varepsilon_{\|} / \varepsilon_{\perp}}$ to identify the hPhPs. As shown in Fig. 3(a), a series of bright bands with strong reflection appear in the two spectral ranges, indicating that multimode $\mathrm{hPhPs}$ waves can be guided by the multilayered structure. 

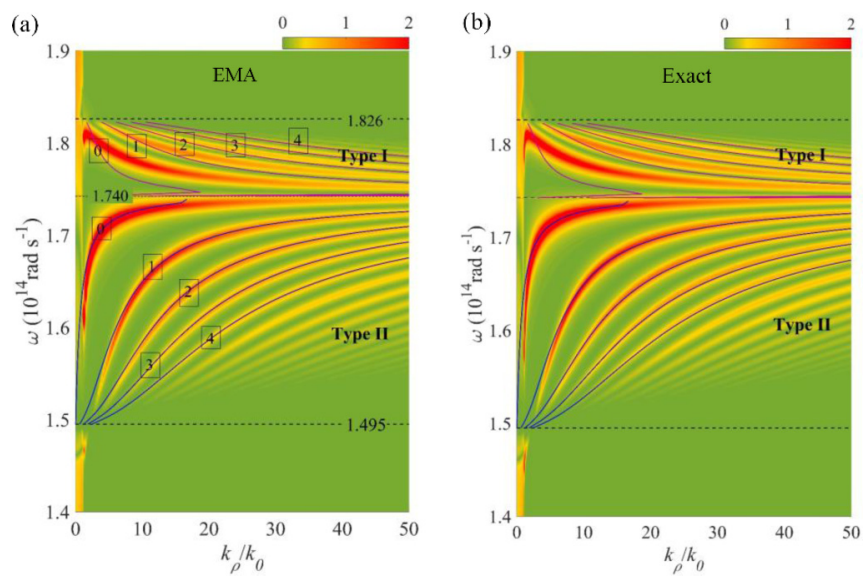

FIG. 3. The 2D distributions of the imaginary part of the transmission coefficient $\operatorname{Im}\left(t_{p}\right)$ of the multilayered structure obtained with (a) EMA and (b) the exact model. Note that $h=1 \mu \mathrm{m}$. The purple and blue lines mark the dispersion relations for type-I and type-II hPhPs, respectively. Integers $0-5$ denote the order of an $\mathrm{hPhP}$ resonance. The frequency limits for the $\mathrm{hPhPs}$ are identified in panel (a).

One can see that the dispersion lines are consistent with the bright bands in $\operatorname{Im}\left(t_{p}\right)$, especially in the type-II region. For comparison, we also present $\operatorname{Im}\left(t_{p}\right)$ obtained with the exact model in Fig. 3(b). The number of layers was set to $N=101$. One can see that the differences between the results from EMA and the exact model are negligible. In addition, we notice that the slope of the dispersion line $(\partial \omega / \partial k)$ in the type-I reststrahlen band is negative, while that in the type-II band is positive. Remarkably, we notice that the slopes of the bright bands in these two zones approach zero at $1.742 \times 10^{14} \mathrm{rad} / \mathrm{s}$, indicating again that the multilayered structure supports the propagation of hPhPs with a very large lateral wave vector at a frequency close to $\omega_{n p}$. We stress that this would be the key feature of our structure with respect to enhancing the RHT between SiC nanoparticles. Our detailed calculations are shown in the next section, and the results confirm this expectation.

In addition to the transmission coefficient, the electric field distribution throughout the multilayered structure is particularly useful for analyzing polariton modes and their associated local field strength increases. Here we adopt a generalized $4 \times 4$ matrix formalism [53,54] for calculating the electric field distribution. SPhPs are excited in the Otto geometry using KRS5 as a highly refractive coupling medium $(n \approx 2.4)$, as shown in Fig. 4(a). Using $p$-polarized light at incidence angles above the critical angle for total internal reflection $\left(\theta_{\text {crit }} \approx 25^{\circ}\right)$ forms evanescent waves in the air gap with large in-plane momentum, which allows coupling to the SPhP modes in the layered structure. The $\vec{E}_{x}$-field amplitudes are shown as a function of frequency and $z$ position at $\theta=80^{\circ}$, and the corresponding critical gap of $d_{\text {gap }}=500 \mathrm{~nm}$ from EMA and the exact model are shown in Figs. 4(b) and 4(c), respectively. In both plots, we see that the $\vec{E}_{x}$ field peaks at $1.742 \times 10^{14} \mathrm{rad} / \mathrm{s}$ and penetrates throughout the multilayered structure, indicating a direct tunneling channel for $\mathrm{hPhP}$ waves near the $\mathrm{SiC}$ nanoparticle resonance $\omega_{n p}$.

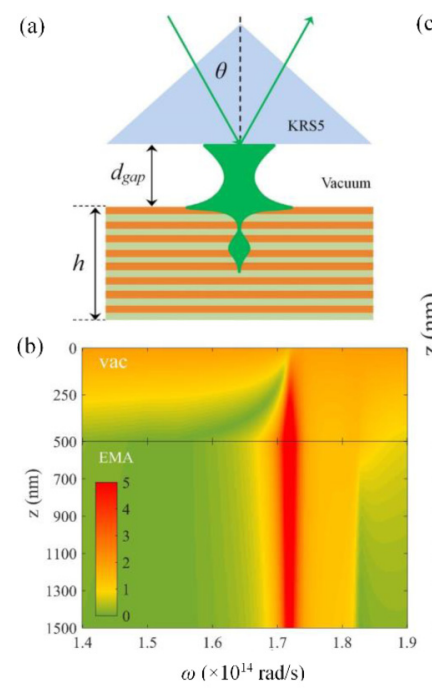

(c)

FIG. 4. Simulated resonances in the multilayered structure with $h=1 \mu \mathrm{m}$ excited in the Otto geometry. (a) Scheme of the Otto geometry using KRS5 as a highly refractive coupling medium $(n \approx$ 2.4). The absolute $\vec{E}_{x}$-field amplitudes from the (b) EMA model and (c) exact model are shown as a function of frequency and $z$ position at $\theta=80^{\circ}$ and the corresponding critical gap of $d_{\text {gap }}=500 \mathrm{~nm}$.

\section{GIANT RESONANT ENHANCEMENT OF RHT BETWEEN NANOPARTICLES}

Up to now, we have analyzed the optical properties of the $\mathrm{SiC} / \mathrm{NaBr}$ multilayered structure. We found that our structure provides a direct tunneling channel for evanescent waves with high- $k$ modes near the SiC nanoparticle resonance $\omega_{n p}$. In this section, we discuss the RHT conductance $\Phi$ at $300 \mathrm{~K}$ between the two $\mathrm{SiC}$ nanoparticles to see how the presence of the multilayered structure affects RHT. The two particles are separated from the intermediate multilayered slab by a distance of $d=50 \mathrm{~nm}$, while the total thickness of the multilayered slab is varied from $100 \mathrm{~nm}$ to $100 \mu \mathrm{m}$, corresponding to distances ranging from near field to far field.

The conductance $\Phi$ between the two SiC nanoparticles at $300 \mathrm{~K}$ for $N=3,11$, or 21 layers is shown in Fig. 5(a). The ratio of $\Phi$ to the conductance in the absence of the structure $\Phi_{0}$ is shown in Fig. 5(b). We also present the results for a single $\mathrm{SiC}$ slab and for the reflection model in Ref. [37]. For a single $\mathrm{SiC}$ slab $(N=1)$, we can also observe an enhancement in RHT due to coupling among evanescent waves at the top and bottom interfaces. However, coupling between surface waves fades gradually as the thickness increases and eventually vanishes when $h=4.0 \mu \mathrm{m}$. A large enhancement in RHT is seen in the presence of multilayered structures $(N>$ 1). More specifically, we observe maximum amplification of $6 \times 10^{3}, 1.33 \times 10^{5}$, and $3.6 \times 10^{5}$ when the thicknesses are $0.95,1.68$, and $1.95 \mu \mathrm{m}$ for $N=3,11$, and 21 , respectively, as shown in Fig. 5(b). The resulting heat transfer in the presence of the multilayered structure with $N=21$ is more than five orders of magnitude higher than that in the absence of the multilayered structure for a given interparticle distance. For $N=21$, the enhancement is two orders of magnitude when the particles are separated by $200 \mathrm{~nm}$, and this amplification persists up to a distance of $38.6 \mu \mathrm{m}$, indicating the 

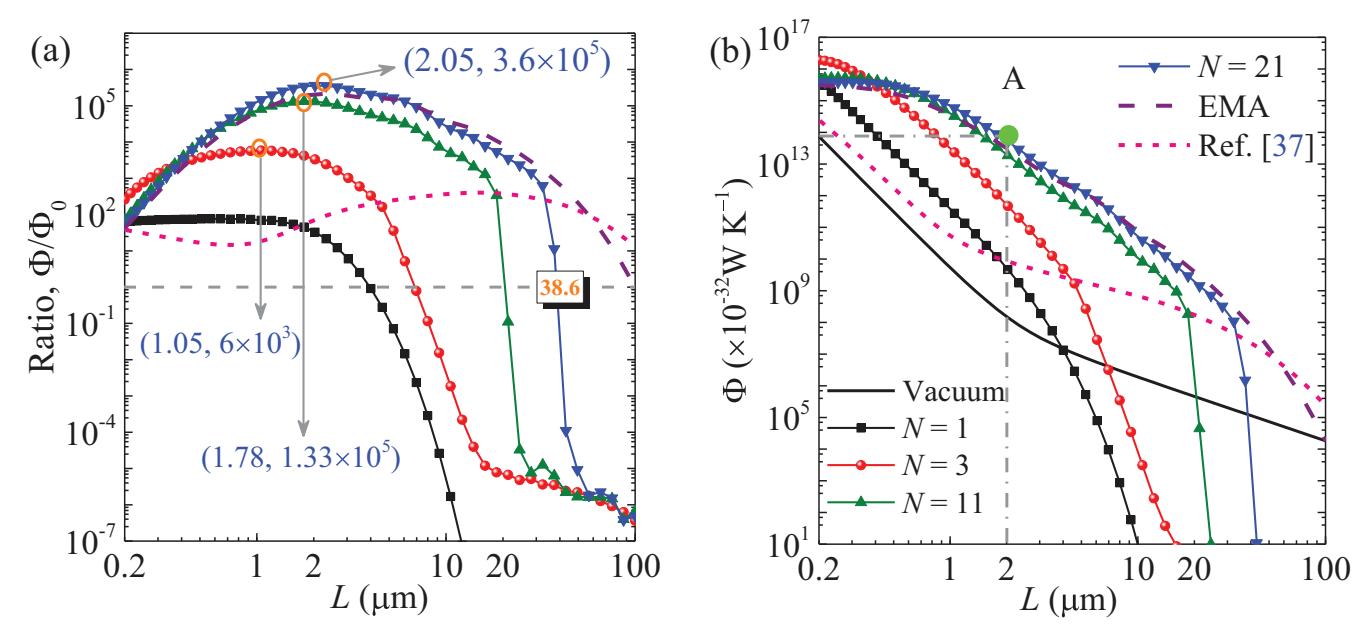

FIG. 5. (a) RHT conductance $\Phi$ between the two particles as a function of interparticle distance $L$. (b) The factor $R$ between $\Phi$ and the conductance in the absence of the structure $\Phi_{0}$. The temperature is $T=300 \mathrm{~K}$. The particles are separated from the surface by a distance $d=50 \mathrm{~nm}$. $h$ is varied from $100 \mathrm{~nm}$ to $100 \mu \mathrm{m}$. Point A in panel (a) denotes the conductance at $2 \mu \mathrm{m}$, which is equal to that at $0.2 \mu \mathrm{m}$ in the absence of the intermediate structure. All slabs are of equal thickness. The symbols/colors in panel (a) mean the same as those in panel (b).

conductance increase persists into the far field. Figure 2(a) shows the conductance at $2 \mu \mathrm{m}$ (point $\mathrm{A}$ ) is equal to that at $0.2 \mu \mathrm{m}$ in the absence of the intermediate structure, which indicates that the same heat flux can be observed in the farfield regime as in the near-field regime.

Because evanescent waves between the adjacent interfaces could not couple with each other when the total thickness is large, RHT from the top particle is invisible to the other side of the slab; hence RHT is suppressed, as shown in Fig. 2. When the two particles are located above the SiC bulk, RHT conductance is amplified by propagation of surface waves [37]. Although direct and reflected RHT between the two particles is included, the maximum amplification is 400 at $20 \mu \mathrm{m}$, which is three orders of magnitude less than the amplification in the presence of our multilayered structure. Note that the above results were obtained using the transmission coefficients of the multilayered structure. Determining the exact solution is time consuming as $N \rightarrow \infty$; thus EMA with $f=0.5$ is used to obtain results when $N \rightarrow \infty$. We find that the EMA results are consistent with those for $N=21$ when $L<40 \mu \mathrm{m}$, and we predict an increase in RHT conductance even when $L$ reaches $100 \mu \mathrm{m}$.

To interpret the underlying physics of the above results and confirm that the frequency-matched high- $k$ modes are indeed responsible for the giant RHT enhancement, we show the conductance spectrum $\Phi_{\omega}$ in Fig. 6(a). The total thickness $h$ was set to $1.95 \mu \mathrm{m}$ for the transmission configuration. We note that $\mathrm{SPhPs}$ in a single $\mathrm{SiC}$ slab or the $\mathrm{SiC}$ bulk exhibit surface resonance at $\omega_{s}=1.786 \times 10^{14} \mathrm{rad} / \mathrm{s}$, which corresponds to the condition $\varepsilon(\omega)+1=0$. We now have three characteristic frequencies in this system, namely, $\omega_{h}, \omega_{n p}$, and $\omega_{s}$, as shown in Fig. 6(a). Notice that the total conductance is larger when $\Phi_{\omega}$ is larger at $\omega_{n p}$, as shown in Fig. 5(a). A second smaller peak emerges at $\omega_{s}$ in the single slab or bulk. However, the contribution to RHT between nanoparticles from this peak is limited. Physically, one can expect that the observed heat flux results from an interaction between waves emitted from the particles and those from the slab or surface. Mathematically, the conductance expressed in Eq. (1) explicitly includes these two waves' characteristics in the polarizability $\alpha$ and the GF, respectively. More specifically, we separate the conductance into $k_{0}^{4}[\operatorname{Im}(\alpha)]^{2}$ and $\operatorname{Tr}\left[\mathcal{G G}^{*}\right]$, as shown in Fig. 6(b). For the bulk or single film, the trace of the GF only peaks at $\omega_{s}$. However, the value of the polarizability at $\omega_{s}$ is far lower than that at $\omega_{n p}$. Hence, as we multiply these two quantities, the contribution from the peak in the GF to amplification is weakened. Meanwhile, due to the very small value of $\operatorname{Tr}\left[\mathcal{G G}^{*}\right]$ at $\omega_{n p}$, the particle resonance's contribution to RHT is also limited. As a result, amplification for a single $\mathrm{SiC}$ nanoparticle is very limited in the transmission and reflection configurations. As a multilayered structure with $N=3$ is used, we see in Fig. 6(a) that an additional peak emerges at a frequency very close to $\omega_{n p}$ in the $\operatorname{Tr}\left[\mathcal{G G}^{*}\right]$ curve, and the peak at $\omega_{s}$ increases to a larger value than that observed from the bulk or single film. When $N=21$, the peak at $\omega_{s}$ in $\operatorname{Tr}\left[\mathcal{G G}^{*}\right]$ nearly disappears due to the increasingly prominent effect of $\mathrm{hPhPs}$, but the other peak is much larger at $\omega_{h}$. Consequently, we obtain a large RHT conductance for $N=21$, as shown in Fig. 6(a), which arises when the resonant frequencies of the nanoparticle and multilayered structure are nearly equal. We call this effect resonant RHT.

To interpret the behavior of $\operatorname{Tr}\left[\mathcal{G G}^{*}\right]$, we show the absolute square of the $z z$ element $\left|G_{z z}\right|^{2}$ as a function of $k_{x}$ and $\omega$ for the bulk and multilayered structure with $N=1$ and 21 in Figs. 6(c)-6(e). One can see that the largest value of the lateral wave number $k_{x}$ for the $\left|G_{z z}\right|^{2}$ contour is truncated. This originates from the evanescent feature of the surface wave. The exponent $e^{i\left[-k_{20} 0\left(z_{2}+h\right)+k_{20} z_{1}\right]}$ in the GF in Eq. (3) introduces a cutoff [41] at $k_{\rho} \approx 1 /(2 d)$. We see that the $\mathrm{SiC}$ bulk supports a single $\mathrm{SiC}$-interface resonance at $\omega_{s}$, which extends to a large lateral wave vector, as shown in Fig. 6(c). For the SiC slab, the evanescent field from the SPhPs associated with each interface interact with each other, leading to a splitting of the $\mathrm{SPhP}$ dispersion relation into antisymmetric (high-frequency) and symmetric (low-frequency) modes, as shown in Fig. 6(d). As the lateral wave number increases, the dispersion relations for both modes asymptotically approach the dispersion curve of a single SiC-vacuum interface, producing a peak in the 

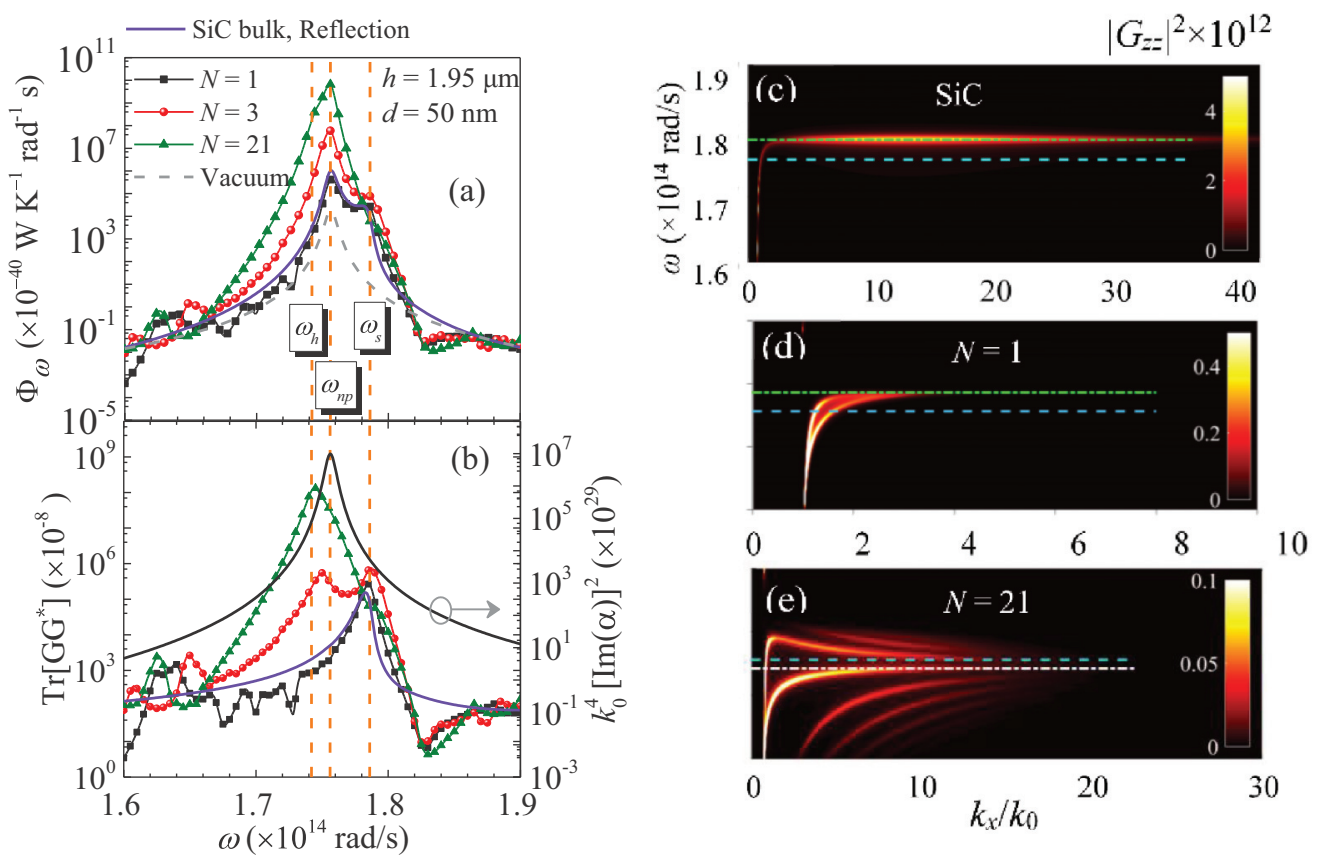

FIG. 6. (a) Spectral conductance $\Phi_{\omega}$. (b) Trace of the GF and polarizability of the SiC particles. The absolute square of the $z z$ element $\left|G_{z z}\right|^{2}$ of (c) the reflection GF at the two positions separated at $L=2.05 \mu \mathrm{m}$ above the SiC bulk, and the transmission GF at the two positions separated by the multilayer structure with (d) $N=1$ and (e) $N=21$ layers. $h=1.95 \mu \mathrm{m}$ for the transmission model and $d=50 \mathrm{~nm}$. The three vertical yellow dotted lines in panels (a),(b) mark the three resonances at $\omega_{h}, \omega_{n p}$, and $\omega_{s}$, respectively. These are shown using horizontal white, blue, and pink dotted lines in panels (c)-(e).

conductance spectrum at $\omega_{s}$ in Fig. 6(a). For a multilayered structure with $N=21,\left|G_{z z}\right|^{2}$ in Fig. 6(e) reveals a similar distribution as that for the transmission coefficient presented in Fig. 3. However, a cut-off wave number is introduced in $\left|G_{z z}\right|^{2}$. The largest $k_{x}$ value for $N=21$ is less than that in the $\mathrm{SiC}$ bulk because resonance at a frequency closer to $\omega_{n p}$ and the hyperbolic dispersion relations are much larger than that of the SiC bulk, as shown in Fig. 6(a).

We note that $f$ is assumed to be 0.5 in the above discussion. Because the optical properties of the multilayered structure depend on $f$, one can expect that $f$ will impact RHT. The RHT conductance with respect to $L$ for $f=0.3,0.5$, and 0.7 determined with EMA is shown in Fig. 7(a). We see that RHT conductance between nanoparticles increases in all cases. At all interparticle distances, the most significant increase is seen when $f=0.5$. When the proportion of $\mathrm{SiC}$ is smaller than that of $\mathrm{NaBr}$, i.e., $f=0.3$, the effect persists up to $L=100 \mu \mathrm{m}$, although amplification is much smaller than the case with $f=0.5$. When $L<5 \mu \mathrm{m}$ the curve for $f=0.7$ lies between that for $f=0.3$ and $f=0.5$, but the $f=0.7$ curve drops below the other curves when $L>5 \mu \mathrm{m}$. For comparison, the exact results with $N=21$ for these three $f$ values are plotted in Fig. 7(a). We find that the results from EMA are consistent with those determined with the exact model when $L \in[1,10] \mu \mathrm{m}$.

To provide insight into the underlying physics, we plot the absolute square of the $z z$ element $\left|G_{z z}\right|^{2}$ in the transmission GF at $L=2.05 \mu \mathrm{m}$ for $f=0.3$ and 0.7 in Figs. 7(b) and 7 (c), respectively. Figure 8(a) shows the dielectric function of the multilayered structure, where we see that two separate hyperbolic bands form when $f=0.3$ or 0.7 , i.e., a type-I band at $\omega \in[1.785-1.826] \times 10^{14} \mathrm{rad} / \mathrm{s}$ and a type-II band at $\omega<$ $1.679 \times 10^{14} \mathrm{rad} / \mathrm{s}$. Indeed, two bright brands within these two bands can be seen in Figs. 7(b) and 7(c), indicating hPhPs features of the multilayered structure. Furthermore, there are many more bright bands in the type-II region than in the type-I region when $f=0.3$. On the contrary, the brightest bands for $f=0.7$ are located in the type-I region. We see that the bright bands in the type I-region are closer to $\omega_{n p}$ than those in the type-II region. Hence, $\Phi$ is larger when the type-I bands in the $G_{z z}$ plot are brighter. Meanwhile, the two bands intersect at $\omega_{h}$ when $f=0.5$, as shown in Figs. 2(c) and 6(e), which significantly increases RHT conductance between particles. One can see that $\Phi_{f=0.3}<\Phi_{f=0.7}<\Phi_{f=0.5}$ at $h=1.95 \mu \mathrm{m}$ in Fig. 7(a). $\Phi$ for $f=0.7$ decreases rapidly as $h$ increases further and eventually decreases below that for $f=0.3$, and even in the absence of the multilayered structure. In fact, the multilayered structure with a large proportion of $\mathrm{SiC}$ behaves more like a single $\mathrm{SiC}$ film, as shown in Fig. 5(a), which can be also confirmed from inspection of Figs. 6(d) and 7(c). At a very large $h$ value, RHT is suppressed due to decoupling of evanescent waves and attenuation of propagating waves near $\omega_{n p}$, as shown in Fig. 7(a).

To provide further insight into the optical characteristics of the multilayered structure, we show a plot of $\left|\varepsilon_{\|}\right| /\left|\varepsilon_{\perp}\right|$ in Fig. 8(b). Two peaks emerge exactly at the edges of the hyperbolic bands when $f=0.3$ and 0.7 . According to the dielectric function curves, the peaks at 1.679 and $1.785 \times$ $10^{14} \mathrm{rad} / \mathrm{s}$ for $f=0.3$ denote the epsilon near pole frequency (ENP) and epsilon near zero (ENZ) frequencies, respectively. Meanwhile, the positions of ENP and ENZ are exchanged when $f=0.7$. In Figs. 7(b) and 7(c), one can see that all 

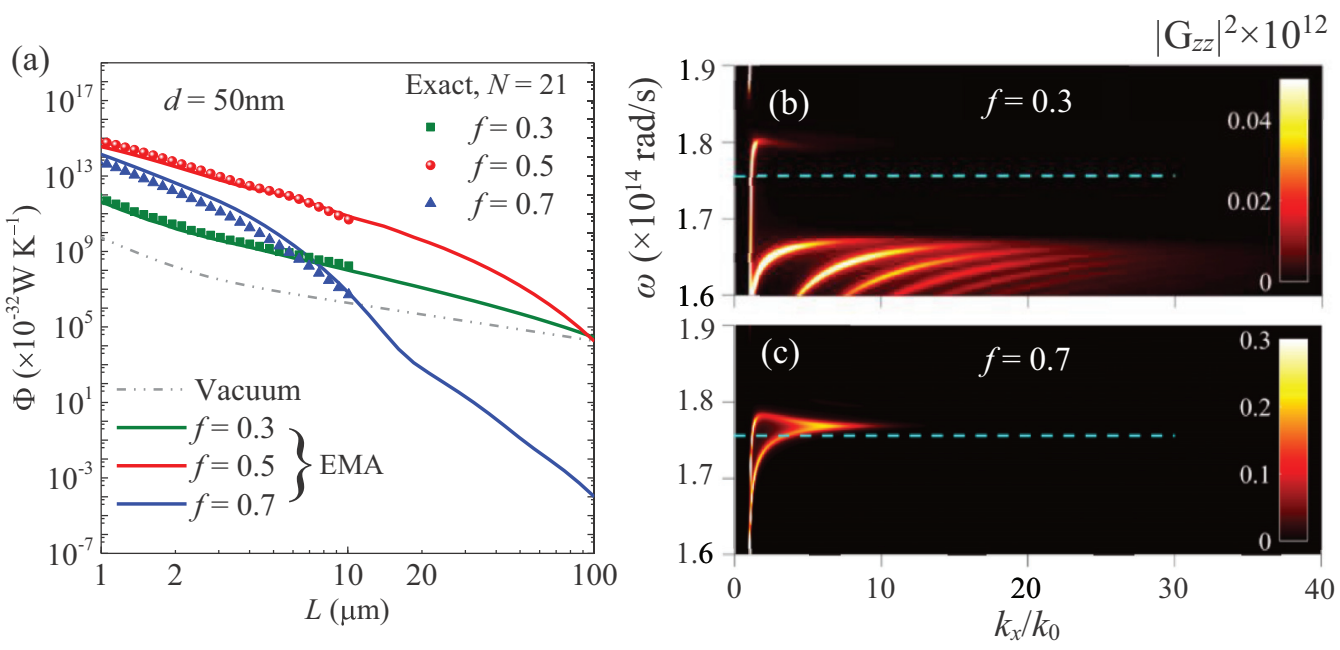

FIG. 7. (a) $\Phi$ obtained using EMA with respect to $L$ in the presence of the multilayer structure with different $f$ values. The absolute square of the $z z$ element in the transmission GF $\left|G_{z z}\right|^{2}$ obtained with EMA for (b) $f=0.3$ and (c) $f=0.7$. In panels (b), (c), the configuration parameters are set to $d=50 \mathrm{~nm}$ and $h=1.95 \mu \mathrm{m}$, and the horizontal blue line marks the resonance at $\omega_{n p}$.

coupled modes converge towards the ENZ and ENP frequencies. Therefore, there are many surface modes with zero group velocity along the interfaces of the multilayered structure at these ENZ and ENP frequencies. Regarding the case where $f=0.5$, ENZ and ENP coincide at $\omega_{h}=1.742 \times 10^{14} \mathrm{rad} / \mathrm{s}$, indicating dominant contributions to RHT conductance at a frequency near the particle resonance. This further confirms

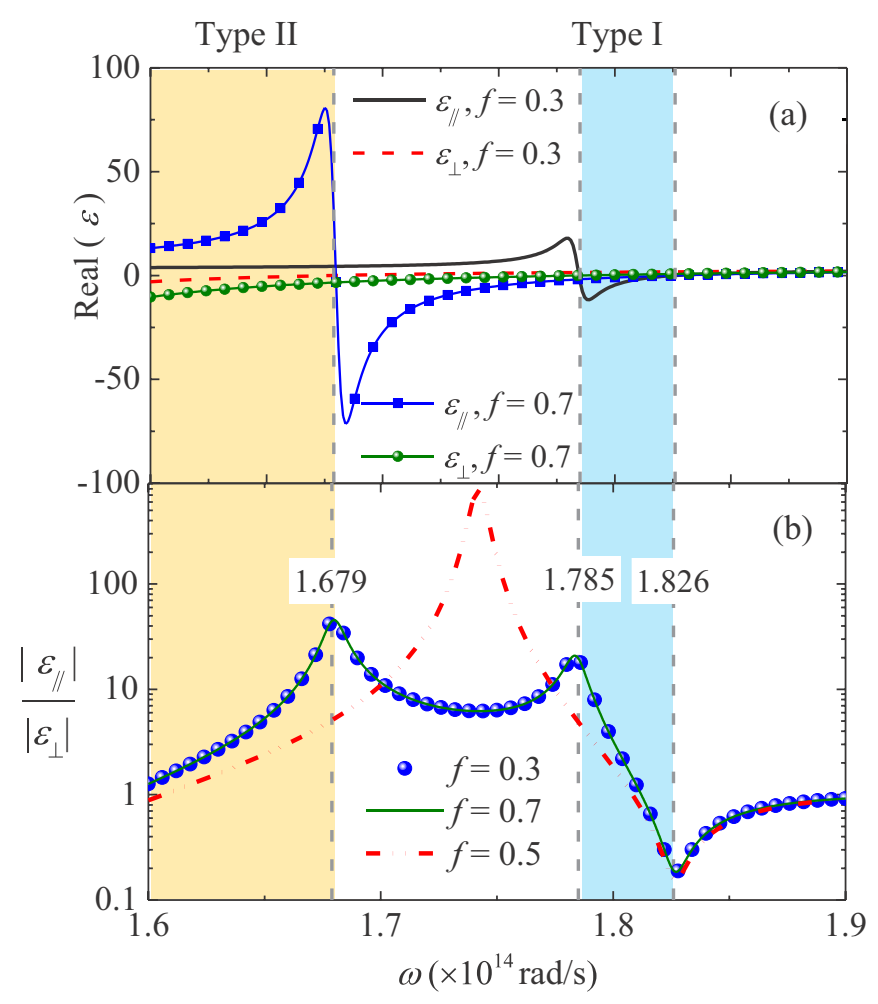

FIG. 8. (a) Dielectric function of the multilayered structure with $f=0.3$ and 0.7 . (b) Plots of $\left|\varepsilon_{\|}\right| /\left|\varepsilon_{\perp}\right|$ with $f=0.3,0.5$, and 0.7 . Type-I and type-II reststrahlen bands are shaded in blue and yellow, respectively. that RHT between nanoparticles can be maximized when $f=0.5$.

\section{OSCILLATING RHT ASSISTED BY THE PROPAGATION OF THE SURFACE WAVES}

Our discussions regarding the transmission configuration have focused on the situation where two nanoparticles lie on the $z$ axis. If we set a lateral distance $d_{x}$ between the two nanoparticles along the $x$ axis, one may expect much more complicated physics than the case with $d_{x}=0$. We stress that the propagation of the evanescent waves along the $x$ axis in addition to the tunneling effects inside the multilayered structure would play a significant role in determining the behavior of RHT between nanoparticles when $d_{x}>0$, which is mathematically included in the term $e^{i k_{x} d_{x}}$ in Eq. (3) and the cylindrical Bessel function in Eq. (5).

Figure 9 shows the total conductance between the two $\mathrm{SiC}$ nanoparticles while the lateral distance $d_{x}$ is varied from $100 \mathrm{~nm}$ to $80 \mu \mathrm{m}$, where $d=50 \mathrm{~nm}$ and $h=1 \mu \mathrm{m}$. The exact model was used to obtain results for $N=1,3$, and 21 with uniform individual layer thickness. EMA results for $f=0.5$ are presented for comparison. We note that $N=21$ is sufficient for convergence when $h=1 \mu \mathrm{m}$. Interestingly, we see that the conductance curves illustrate the oscillating and nonmonotonic decreasing trends with respect to $d_{x}$. Physically, we stress that these undulations originate from interference between surface waves inside the multilayered structure [55].

Because the intermediate structure provides many surface modes when $\omega_{h}=1.742 \times 10^{14} \mathrm{rad} / \mathrm{s}$, the trace of the GF exhibits a peak at $\lambda_{h} \approx 10.82 \mu \mathrm{m}$, as shown in Fig. 6(b), while after the product between the $\operatorname{Tr}\left[\mathcal{G G}^{*}\right]$ and $k_{0}^{4}[\operatorname{Im}(\alpha)]^{2}$, the peak conductance is found at $\lambda_{n p} \approx 10.75 \mu \mathrm{m}$ (corresponding to the nanoparticle resonance). Figure 9(b) shows that the periodicity of the fringes is $5.375 \pm 0.3 \mu \mathrm{m}$, which is approximately half of $\lambda_{n p}$. Figure 9(c) intuitively shows the oscillating behavior of the spectral conductance between the 
(a)

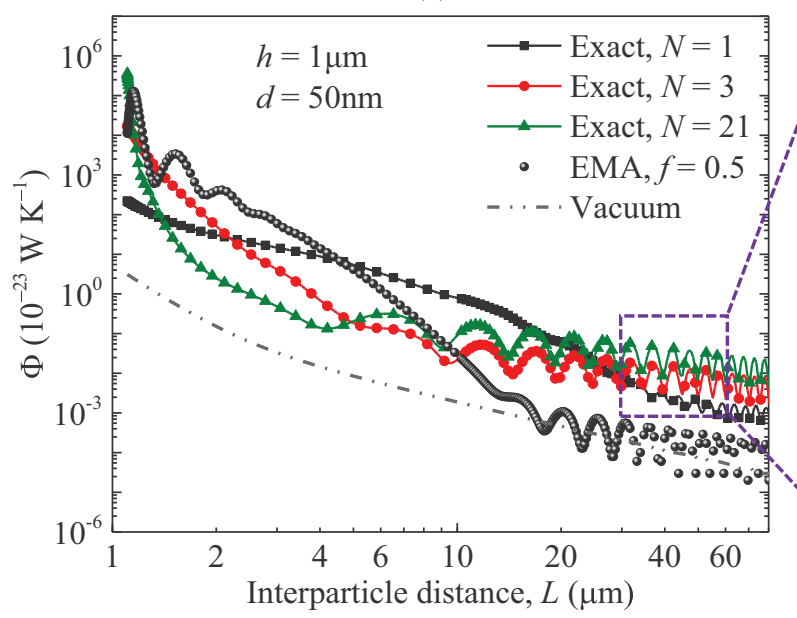

(b)

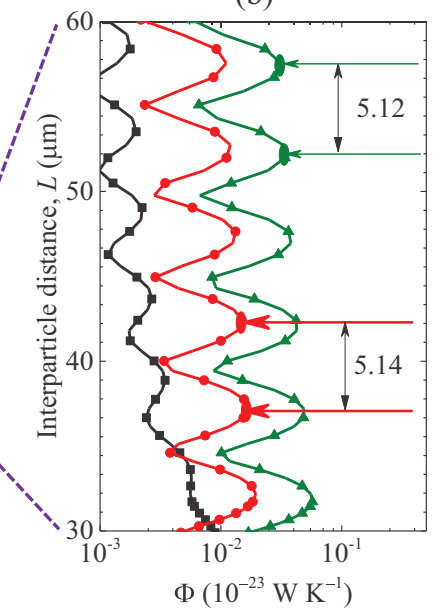

(c)

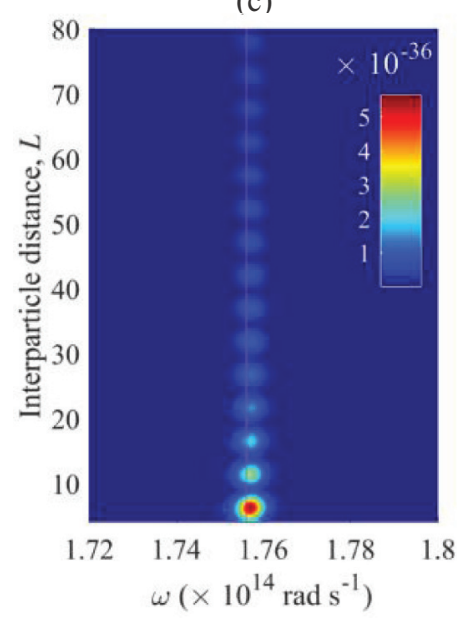

FIG. 9. (a) RHT conductance with respect to the interparticle distance in the multilayered structure with 1, 3, and 21 layers when the lateral distance $d_{x}$ is varied from $100 \mathrm{~nm}$ to $80 \mu \mathrm{m}$. The particle-surface distance $d=50 \mathrm{~nm}$ and the total thickness is $h=1 \mu \mathrm{m}$. (b) Partially magnified view of panel (a). (c) RHT conductance spectrum between the two particles in the presence of multilayered $\mathrm{SiC} / \mathrm{NaBr}$ with $N=21$. The red line in panel (c) denotes the nanoparticle resonance.

two particles in the presence of the multilayered structure with $N=21$. Evanescent wave tunneling dominates heat transfer when $d_{x}$ is small. Because there are many surface modes near the nanoparticle frequency, a larger conductance is seen when $N$ is larger; i.e., $\Phi_{N=21}>\Phi_{N=3}>\Phi_{N=1}$. Wave propagation plays an increasingly prominent role as $d_{x}$ increases. Mathematically, the effects of $d_{x}$ are manifested in the term $k_{\rho} d_{x}$ in the argument of the Bessel function in Eq. (1). Figures 10(a)-10(c) show $\left|G_{z z}\right|^{2}$ in the transmission GF with $L=1.1 \mu \mathrm{m}\left(d_{x}=0\right), 3 \mu \mathrm{m}\left(d_{x}=2.791 \mu \mathrm{m}\right)$, and $40 \mu \mathrm{m}$ $\left(d_{x}=39.985 \mu \mathrm{m}\right)$, respectively. For $d_{x}=0$ in Fig. 10(a), the GF distribution is continuous along the $k_{\rho}$ axis, whereas the GF exhibits an oscillating behavior with respect to $k_{\rho}$ when $d_{x}>0$. Moreover, as $d_{x}$ increases, the periodicity of the fringes decreases; hence the high-frequency oscillation can be seen in Fig. 10(c). However, the value of the GF decreases as $d_{x}$ increases. Physically, this trend coincides with fact that the amplitude of the surface waves decreases exponentially towards the propagation direction.

Let us interpret the trends shown in Fig. 9(a). The RHT conductance decreases at a faster rate as $d_{x}$ increases when $N$ is larger. The relationship $\Phi_{N=21}>\Phi_{N=3}>\Phi_{N=1}$ reverses to $\Phi_{N=21}<\Phi_{N=3}<\Phi_{N=1}$ when $d_{x}=2.791 \mu \mathrm{m}$. This is owing to the fact that an oscillation with large periodicity in the GF weakens the advantage coming from the many modes for a large $N$. However, at a large $d_{x}$, since the oscillating periodicity in the GF becomes very small as shown in Fig. 10(c), the scenario approaches that shown in Fig. 10(a). As a result, the advantage of using a large $N$ value is reproduced and the relation $\Phi_{N=21}>\Phi_{N=3}>\Phi_{N=1}$ emerges again. Moreover, we observe that the oscillating behavior emerges earlier when $N$ is larger. This is because many more surface waves interfere when $N$ is larger. In addition, a comparison of the EMA results with those from the exact model for $N=21$ shows that EMA is not suitable for predicting the RHT conductance when there is a lateral distance between the two particles.

Figure 11 shows the RHT conductance when $h=5$ or $10 \mu \mathrm{m}$ and as $d_{x}$ is varied from $100 \mathrm{~nm}$ to $1000 \mu \mathrm{m}$. It is obvious that $\Phi$ for $N=21$ is larger than that for $N=1$. An oscillating behavior on the curve can be seen in Fig. 11 at a larger $d_{x}$ than that for $h=1 \mu \mathrm{m}$, as shown in Fig. 9(a). Moreover, for the multilayered structure with large $N$, both the amplitude and periodicity of the fringes are larger than those for a single SiC film. This oscillation in RHT is an example of an electromagnetic resonator in the context of thermal radiation [38]. Based on Fig. 11, one can expect that (a)

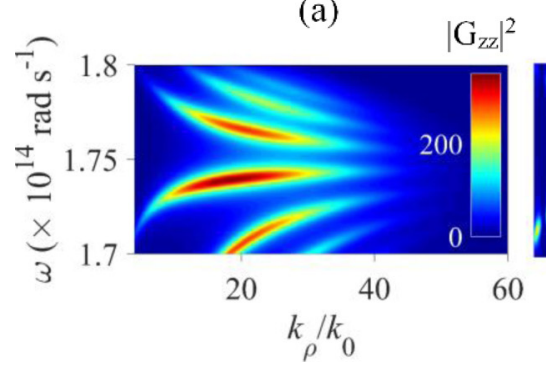

(b)

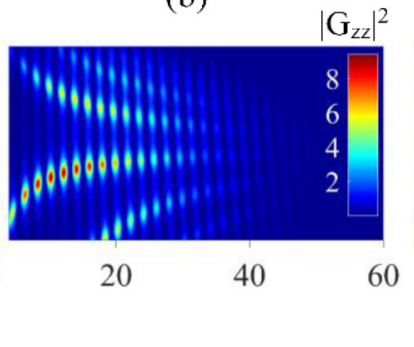

(c)

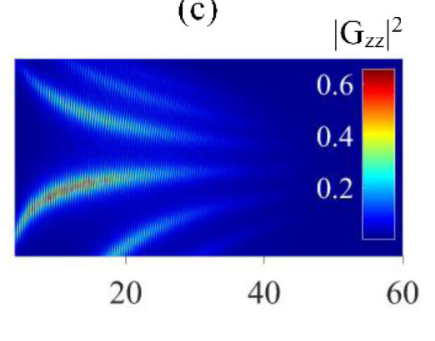

FIG. 10. $\left|G_{z z}\right|^{2}$ in the transmission GF for (a) $L=1.1 \mu \mathrm{m}\left(d_{x}=0\right)$, (b) $L=3 \mu \mathrm{m}\left(d_{x}=2.791 \mu \mathrm{m}\right)$, and (c) $L=40 \mu \mathrm{m}\left(d_{x}=39.985 \mu \mathrm{m}\right)$. $h=1 \mu \mathrm{m}$ and $N=21$. 


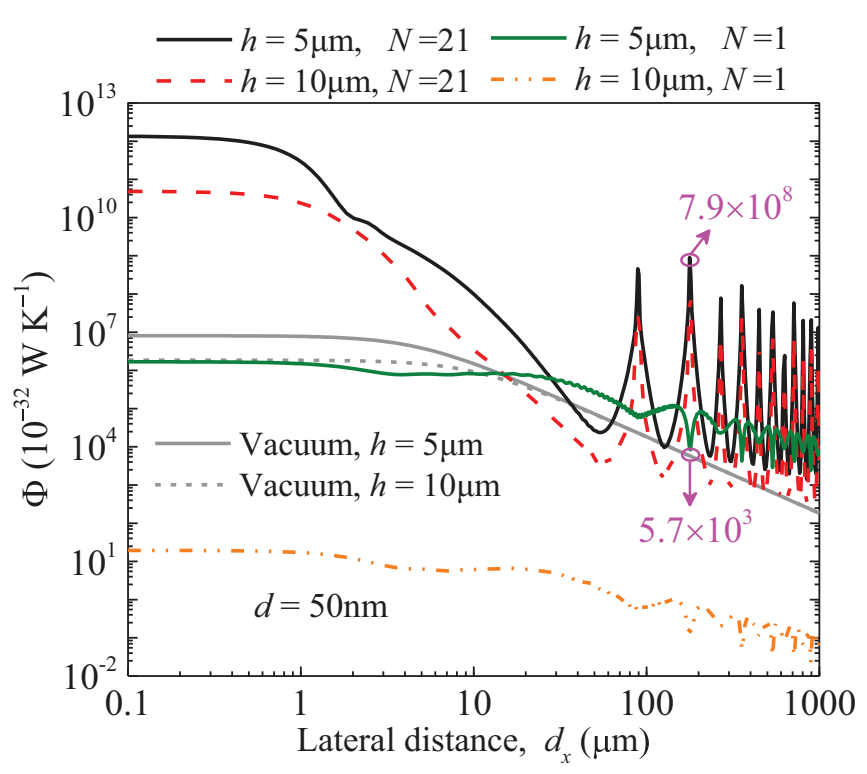

FIG. 11. RHT conductance with respect to $d_{x}$ for a multilayered structure with $N=1$ or $21, d=50 \mathrm{~nm}$, and $h=5$ and $10 \mu \mathrm{m}$.

the giant increase in RHT conductance could be also seen in the very far-field regime, for instance, for $h=5 \mu \mathrm{m}$ and $N=21$ at $d_{x}=176.9 \mu \mathrm{m}$, where the amplification factor is $1.386 \times 10^{5}$.

\section{CONCLUSIONS}

RHT between two nanoparticles in the presence of a periodic multilayered structure with alternating materials was examined in this study. Remarkably, the edge frequencies of the type-I and type-II hyperbolic bands in this structure coincide at a frequency that is approximately equal to the particle resonance, indicating high- $k$ modes resonate in phase with the particle. We studied how radiative heat is exchanged between nanoparticles when they are placed on each side of the multilayered structure. Due to the frequency matching between the multilayered structure and the nanoparticles, giant resonant RHT between nanoparticles can be observed with an amplification factor of more than five orders of magnitude. The effects of changes in the number of layers was analyzed in the frequency domain, and the results highlight the role played by the hyperbolic waveguides. Furthermore, we found that the largest RHT conductance amplification is seen due to coincidence between the epsilon near pole and epsilon near zero frequencies when the fill factor is 0.5. Finally, we studied RHT when there is a lateral distance between nanoparticles. Due to interference between surface waves, an oscillating nonmonotonic behavior in the RHT with respect to the lateral distance was observed. These findings illustrate one way to regulate energy transfer in systems of particles and could be used to considerably improve the efficiency of nano-micro energy conversion devices.

The present study could be naturally extended to examine RHT along a chain of nanoparticles or nanoparticle clusters mediated by the intermediate slab, as well as RHT in nonreciprocal dispersive systems where unidirectional heat transfer becomes possible.

\section{ACKNOWLEDGMENTS}

This work was supported by the National Natural Science Foundation of China (Grant No. 51706053), as well as the Fundamental Research Funds for the Central Universities (Grant No. HIT.NSRIF. 201842), and by the China Postdoctoral Science Foundation (Grant No. 2017M610208). M.A. acknowledges support from the Institute Universitaire de France, Paris, France (UE).
[1] D. Polder and M. Van Hove, Phys. Rev. B 4, 3303 (1971).

[2] M. Planck, The Theory of Heat Radiation (Dover, New York, 1991).

[3] Y. Yang and L. P. Wang, Phys. Rev. Lett. 117, 044301 (2016).

[4] V. Fernández-Hurtado, F. J. García-Vidal, S. H. Fan, and J. C. Cuevas, Phys. Rev. Lett. 118, 203901 (2017).

[5] G. M. Tang, H. H. Yap, J. Ren, and J. S. Wang, Phys. Rev. Appl. 11, 031004 (2019).

[6] J. Yang, W. Du, Y. S. Su, Y. Fu, S. X. Gong, S. L. He, and Y. G. Ma, Nat. Commun. 9, 4033 (2018).

[7] J. D. Shen, X. L. Liu, and Y. M. Xuan, Phys. Rev. Appl. 10, 034029 (2018).

[8] Y. Zhang, H. L. Yi, and H. P. Tan, ACS Photonics 5, 3739 (2018).

[9] K. Joulain, J. P. Mulet, F. Marquier, R. Carminati, and J. J. Greffet, Surf. Sci. Rep. 57, 59 (2005).

[10] S. Basu, Z. M. Zhang, and C. J. Fu, Int. J. Energy Res. 33, 1203 (2009).

[11] S. Shen, A. Narayanaswamy, and G. Chen, Nano Lett. 9, 2909 (2009).

[12] K. Kim, B. Song, V. Fernandez-Hurtado, W. Lee, W. Jeong, L. Cui, D. Thompson, J. Feist, M. T. Homer Reid, F. J. Garcia-
Vidal, J. C. Cuevas, E. Meyhofer, and P. Reddy, Nature 528, 387 (2015).

[13] K. Ito, A. Miura, H. Iizuka, and H. Toshiyoshi, Appl. Phys. Lett. 106, 083504 (2015).

[14] M. P. Bernardi, D. Milovich, and M. Francoeur, Nat. Commun. 7, 12900 (2016).

[15] A. Kittel, W. Muller-Hirsch, J. Parisi, S.-A. Biehs, D. Reddig, and M. Holthaus, Phys. Rev. Lett. 95, 224301 (2005).

[16] Y. Zhang, C. H. Wang, H. L. Yi, and H. P. Tan, J. Quantum Spectrosc. Radiat. Transfer 221, 138 (2018).

[17] M. J. He, H. Qi, Y. F. Wang, Y. T. Ren, W. H. Cai, and L. M. Ruan, Opt. Express 27, A953 (2019).

[18] H. H. Wu, Y. Huang, L. J. Cui, and K. Y. Zhu, Phys. Rev. Appl. 11, 054020 (2019).

[19] D. T. Debu, M. H. Doha, H. Churchill, and J. B. Herzog, RSC Adv. 9, 29173 (2019).

[20] J. Song, M. Lim, S. S. Lee, and B. J. Lee, Phys. Rev. Applied 11, 044040 (2019).

[21] A. Narayanaswamy and G. Chen, Appl. Phys. Lett. 82, 3544 (2003).

[22] W. Srituravanich, N. Fang, C. Sun, Q. Luo, and X. Zhang, Nano Lett. 4, 1085 (2004). 
[23] P. Ben-Abdallah and S.-A. Biehs, AIP Adv. 5, 053502 (2015).

[24] P. Ben-Abdallah and S.-A. Biehs, Phys. Rev. Lett. 112, 044301 (2014).

[25] K. Ito, K. Nishikawa, and H. Iizuka, Appl. Phys. Lett. 108, 053507 (2016).

[26] C. R. Otey, W. T. Lau, and S. Fan, Phys. Rev. Lett. 104, 154301 (2010).

[27] H. Iizuka and A. Fan, J. Appl. Phys. 112, 024304 (2012).

[28] J. Huang, Q. Li, Z. Zheng, and Y. Xuan, Int. J. Heat Mass Transfer 67, 575 (2013).

[29] K. Ito, K. Nishikawa, H. Iizuka, and H. Toshiyoshi, Appl. Phys. Lett. 105, 253503 (2014).

[30] V. Kubytskyi, S.-A. Biehs, and P. Ben-Abdallah, Phys. Rev. Lett. 113, 074301 (2014).

[31] R. Messina, M. Antezza, and P. Ben-Abdallah, Phys. Rev. Lett. 109, 244302 (2012).

[32] R. Messina, P. Ben-Abdallah, B. Guizal, M. Antezza, and S.-A. Biehs, Phys. Rev. B 94, 104301 (2016).

[33] P. Ben-Abdallah, S.-A. Biehs, and K. Joulain, Phys. Rev. Lett. 107, 114301 (2011).

[34] R. Messina and M. Antezza, Phys. Rev. A 89, 052104 (2014).

[35] K. Asheichyk, B. Müller, and M. Krüger, Phys. Rev. B 96, 155402 (2017).

[36] J. Dong, J. Zhao, and L. Liu, Phys. Rev. B 97, 075422 (2018).

[37] R. Messina, S.-A. Biehs, and P. Ben-Abdallah, Phys. Rev. B 97, 165437 (2018).

[38] K. Asheichyk and M. Kruger, Phys. Rev. B 98, 195401 (2018).

[39] Y. Zhang, M. Antezza, H. L. Yi, and H. P. Tan, Phys. Rev. B 100, 085426 (2019).
[40] A. Ott, R. Messina, P. Ben-Abdallah, and S.-A. Biehs, Appl. Phys. Lett. 114, 163105 (2019).

[41] P. Andrew and W. L. Barnes, Science 306, 1002 (2004).

[42] S.-A. Biehs, V. M. Menon, and G. S. Agarwal, Phys. Rev. B 93, 245439 (2016).

[43] R. Deshmukh, S.-A. Biehs, E. Khwaja, T. Galfsky, G. S. Agarwal, and V. M. Menon, ACS Photonics 5, 2737 (2018).

[44] B. Zhao, B. Guizal, Z. M. Zhang, S. H. Fan, and M. Antezza, Phys. Rev. B 95, 245437 (2017).

[45] C. Abbas, B. Guizal, and M. Antezza, Phys. Rev. Lett. 118, 126101 (2017).

[46] M. Lim, J. Song, and S. S. Lee, and B. J. Lee, Nat. Commun. 9 , 4302 (2018).

[47] H. Iizuka and S. H. Fan, Phys. Rev. Lett. 120, 063901 (2018).

[48] L. Novotny and B. Hecht, Principles of Nano-optics (Cambridge University Press, Cambridge, 2012).

[49] K. Shi, F. Bao, and S. He, ACS Photonics 4, 971 (2017).

[50] Z. M. Zhang, Nano/Microscale Heat Transfer (McGraw-Hill, New York, 2007).

[51] Handbook of Optical Constants of Solids, edited by E. Palik (Academic, New York, 1998).

[52] J. E. Pérez-Rodríguez, G. Pirruccio, and R. Esquivel-Sirvent, Phys. Rev. Mater. 3, 015201 (2019).

[53] N. C. Passler and A. Paarmann, https://doi.org/10.5281/zenodo. 847015 (2017).

[54] N. C. Passler and A. Paarmann, J. Opt. Soc. Am. B 34, 2128 (2017).

[55] P. Johansson, Phys. Rev. B 83, 195408 (2011). 